\title{
Prevalence of ear cleaning and its characteristic physical findings in patients visiting tertiary care center in Bhutan
}

\author{
Tika R. Adhikari ${ }^{1}$, Sonam Jamtsho
}

${ }^{1,2}$ Department of Otorhinolaryngology, Jigme Dorji Wangchuk National Refferal Hospital, Thimphu, Bhutan

\begin{abstract}
Introduction: Many studies have been done on prevalence and complications of ear bud across the globe but very few talk about the characteristics physical findings associated with ear cleaning. We aim to find the prevalence and characteristic physical findings of cotton bud usage in patients visiting a tertiary care center in Bhutan. Methods: A cross sectional study in a tertiary care center in Bhutan over a period of one month. Results: The prevalence of ear cleaning was $92.15 \%$. The most common physical finding was shiny external auditory canal (66\%), excoriations in the external auditory canal (longitudinal furrowing along the floor or Circular scratch marks) (52\%), erythema and edema of ear canal (46.6\%), wax in the bony canal near tympanic membrane (9.3\%), stenosed external auditory canal (3.8\%), cotton wool remnants in ear canal (2.8\%).Conclusions: It is important to recognize these characteristic clinical findings of cleaning the ear so that proper counseling can be given against its usage and prevent undue complications associated with it.
\end{abstract}

Key Words: Cotton bud; Complications; Prevalence.

\section{INTRODUCTION}

Cerumen or wax is defined as a mixture of secretions (sebum together with secretions from modified apocrine sweat glands) and sloughed epithelial cells, and is a normal substance present in the external auditory canal ${ }^{1}$. It is expelled by epithelial migration from the tympanic membrane, aided by movements of the temporomandibular joint thus rendering the ear a self-cleansing mechanism. However, people are ignorant about this facts and prevalence of ear cleaning is reported as high as over $90 \%^{2,3}$. Use of cotton bud is not only unnecessary but potentially dangerous. Ear injuries caused by the use of cotton bud are commonly seen in ear, nose and throat(ENT) practice ${ }^{4}$. It can lead to infection, retention of the cotton bud, wax plug impaction, with resultant discomfort, deafness and vertigo, injury to the external auditory canal or perforation of the tympanic membrane, facial nerve paralysis and inner ear damage.

Many studies have been done on prevalence and complications of ear bud across the globe but very few talk about the characteristics physical findings associated with ear cleaning. It is important to recognize these characteristic clinical findings of cotton bud usage so that appropriate counseling can be given against its usage. This has become more important due to the fact that people deny cleaning ear canal inspite of doing it regularly probably due to stigma. In this study, we aim to find the prevalence of cotton bud usage in patients visiting tertiary care

\section{Corresponding author:}

Tika R. Adhikari

tikaram78@gmail.com center in Bhutan, the frequency, mode and reason for using it. Secondly to find the characteristic physical findings associated with cotton bud use and sharing the findings with other health care workers so that they can advise against the use of cotton bud and prevent complications associated with it.

\section{METHODS}

Study Design: A cross-sectional study was conducted at Jigme Dorji Wangchuck National Referral Hospital (JDWNRH) over a period of one month from $1^{\text {st }}$ May, 2017 to $30^{\text {th }}$ May, 2017 to estimate the prevalence of use of cotton buds and other objects for cleaning ear, and characterize their associated lesions. Patients presenting with non-ear symptoms in Outpatient Department (OPD) were randomly sampled using systematic random sampling technique. Every day approximately 25 individuals were recruited for 20 days spread over a one-month period. Only individual providing written consent and presenting with the non-ear problem were recruited in the study. For minors, written consent and assent was obtained. People with coexistent ear disease were excluded from the study.

Ethical concerns: The study was conducted with prior approval from Research Ethical Board of Bhutan (REBH)(REBH/ Approval/2017/012). Approval was also sought from the Hospital administration for the conduct of the study.

Sample size: The number of samples size required for the estimation of prevalence of cotton bud use was estimated based on the following formula: 


$$
n=\frac{\mathrm{z}_{\mathrm{a} / 2}{ }^{2} \mathrm{pq}}{\mathrm{d}^{2}}
$$

$p=$ expected proportion of cotton bud usage

$q=1-p$

$d=$ Margin of error in estimating $\mathrm{p}$ or maximum absolute error

$\alpha=$ probability of type I error, wheretaking $50 \%$ prevalence(other countries showed 50-90\%), error $4 \%$

$1.96 \times 1.96 x .5 \times .5 / .04 x .04=600$

To make up for losses and missing information (approximately estimated to $10 \%$ ), the sample size required for the study is rounded to 650 people.

The patients were given structured questionnaire to fill. Demographic data, educational level, the perception of ear cleaning, frequency, reason, and mode of ear cleaning were recorded. Those who cleaned their ears were subjected to physical examination using otoscopy and microscopy. The different findings associated with ear cleaning were recorded. In addition standard pure tone audiometry and tympanometry were performed by the audiologist where indicated.

\section{RESULTS}

A total of 650 participants were recruited in the study (344 female and 306 male). $87.85 \%$ had the perception that ear cleaning was good for their health which was consistent with the high prevalence of ear cleaning. The prevalence of ear cleaning was $92.15 \%$ although most of them said they cleaned sometimes only. Figure 1a shows the distribution of participants by education level and frequency of ear cleaning. The prevalence of ear cleaning was high irrespective of educational level. The frequency of ear cleaning is depicted in Figure 1b. The different reasons cited for using cotton buds were itchiness (59.29\%), after bath (16.25\%), discharge $(7.43 \%)$, habit $(7.28 \%)$, ear pleasure $(4.80 \%)$, clean wax $(3.10 \%)$, advice by friends and family $(1.70 \%)$ and advice by health staff $(0.15 \%)$. There were $6.15 \%$ of participants who said they underwent cleaning by quacks in streets. Although majority said they used cotton bud (Q-tips) for cleaning ear, there were quite a few who used dangerously sharp objects like matchsticks, pin or anything that comes in hand (Figure 2).

Physical examination with otoscopy and microscopy were carried out in 599 of the total of 650 participants who had the history of ear cleaning. The different findings were in isolation or in the combination of different findings. The most common finding was shiny external auditory canal (66\%), excoriations in the external auditory canal (longitudinal furrowing along the floor or Circular scratch marks) (52\%), erythema and edema of ear canal $(46.6 \%)$, wax in the bony canal near tympanic

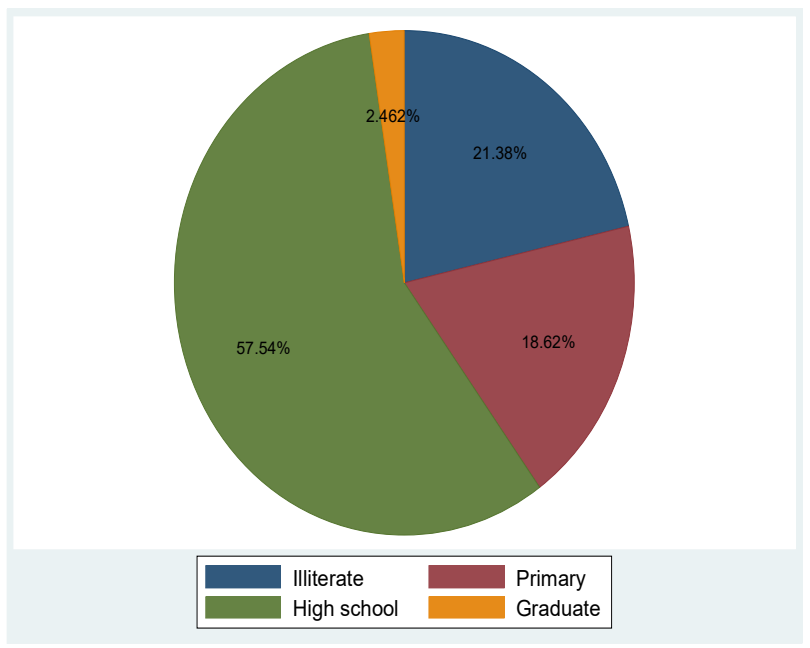

Figure 1a. Distribution of participants by education level

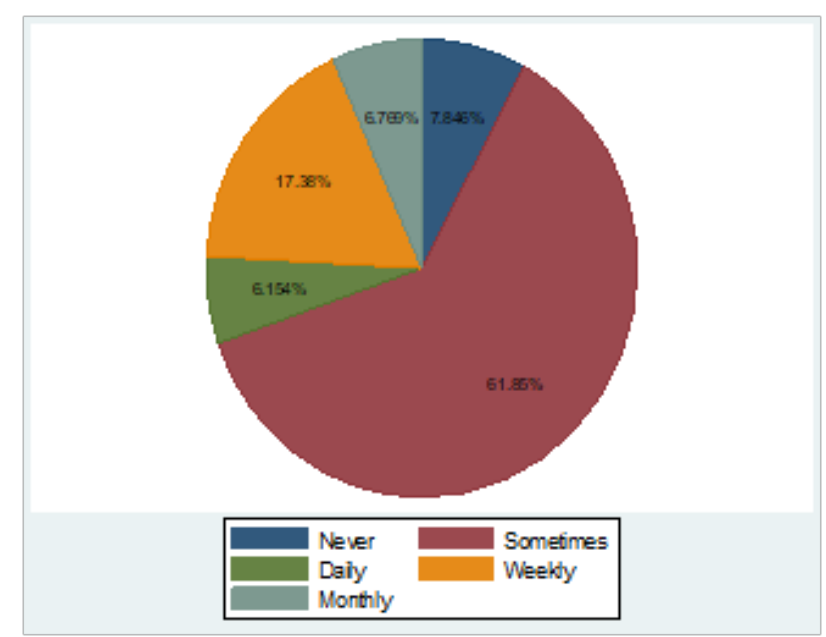

Figure 1b. Frequency of Ear cleaning among participants among different participants

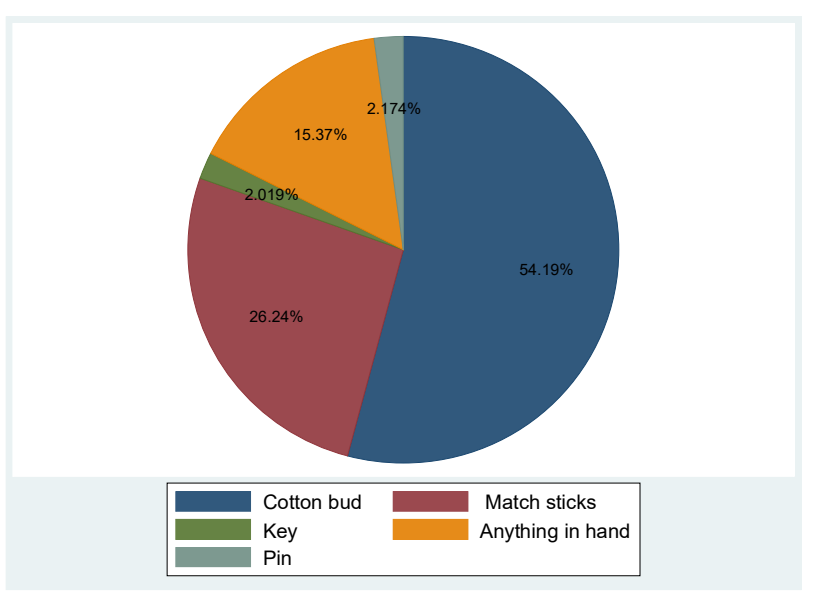

Figure 2. Pie chart to show different objects used for ear cleaning by the participants 
membrane $(9.3 \%)$, stenosed external auditory canal (3.8\%), cotton wool remnants in ear canal(2.8\%). Some of the findings were overlapping (Figure 3a,3b,3c).

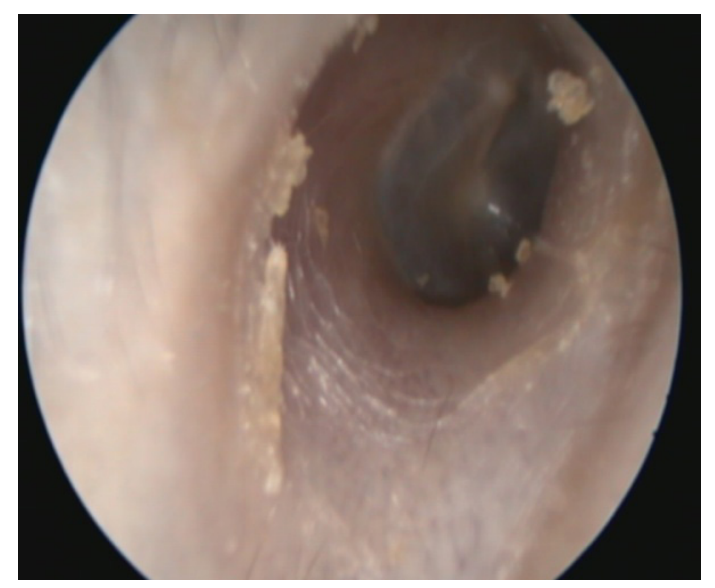

Figure 3a. Circular marks and flaking seen in patients who clean their ear

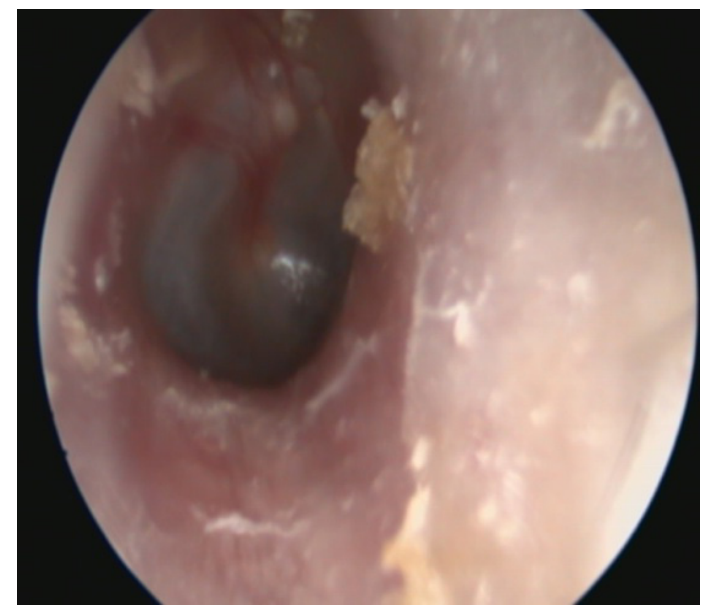

Figure 3b. Longitudinal furrow, erythema, edema, shiny canal in ear cleaners

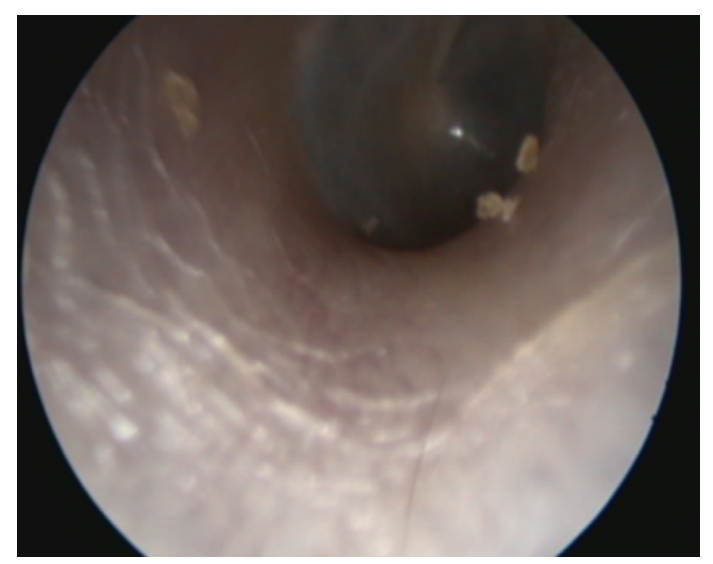

Figure 3c. Physical findings of wax in medial bony canal and circular nail marks in ear cleaners

\section{DISCUSSIONS}

Cerumen or wax is defined as the mixture of secretions (sebum together with secretions from modified apocrine sweat glands) and sloughed epithelial cells, and is a normal substance present in the external auditory canal. It serves to protect, clean, and lubricate the skin of the ear canal.It is expelled by epithelial migration from the tympanic membrane, aided by movements of the temporomandibular joint.Thus ear has a self- cleansing mechanism. Cotton buds are commonly used to clean the ears, remove wax, in case of itching in the ear, aural toilet in discharging ear and as a habit. In a study done by Jonathan C Hobson, the reasons given by patients for using cotton buds in ear canal were advertisements, advice by doctor or nurses, because their family and friends use them, because it seemed like a good idea or they were not sure of the reasons ${ }^{4}$. In our study the reason for cleaning ear werecited as itchiness $(59.29 \%)$, after bath (16.25\%), discharge(7.43\%), as a habit(7.28\%), for ear pleasure(4.80\%), to clean $\operatorname{wax}(3.10 \%)$, advice by friends and family $(1.70 \%)$ or advice by health staffs $(0.15 \%)$.Cleaning of the ear canal is not only unnecessary but also potentially dangerous. Ear injuries caused by use of cotton bud are commonly seen in ENT practice $e^{4,5}$. Cotton bud use may give rise to some dangerous complications ${ }^{6}$. It is unnecessary and a dangerous practice. Unwise efforts with a cotton bud can produce infection, retention of cotton bud, wax plug impaction, with resultant discomfort, deafness and vertigo, injury to the external auditory canal or perforation of the tympanic membrane ${ }^{7}$. Otitis externa can likewise be caused by the overzealous use of cotton buds. Nussinovitch $\mathrm{M}$ et al. found use of cotton tip as the leading cause of otitis externa ${ }^{8}$. Serious complications such as ossicular chain damage, facial nerve palsy, inner ear penetration have been reported by Smith $\mathrm{M}_{\text {et }} \mathrm{al}^{9}$.

Cerumen impaction is present in approximately ten percent of children, five percent of normal healthy adults, up to 57 percent of older patients in nursing homes, and 36 percent of patients with mental retardation ${ }^{10}$.An anatomic deformity and an increased number of hairs in the external auditory canal, as well as physical barriers to natural wax extrusion (e.g. cotton swabs, hearing aids, earplug-type hearing protectors) have been associated with an increased incidence of cerumen impaction. It is usually asymptomatic but when it becomes impacted it can cause complications such as hearing loss, pain, dizziness, or itching. It can also interfere with the examination of the tympanic membrane. Cerumen removal is the most common ENT procedure performed in primary care. It needs removal only in symptomatic patients and if it interferes with examination of ear.

Shamim Ahmed et al in Pakistan ${ }^{6}$ and Kravitz et al ${ }^{11}$ suggested characteristic signs associated with cleaning the ear. In our study we found the signs of cotton bud usage such as shiny external auditory canal (66\%), excoriations in the external auditory canal (longitudinal furrowing along the floor or Circular scratch marks) (52\%), erythema and edema of ear canal (46.6\%), 
wax in the bony canal near tympanic membrane $(9.3 \%)$, stenosed external auditory canal (3.8\%), cotton wool remnants in ear canal $(2.8 \%)$. It has been observed that Bhutanese patients tend to deny ear cleaning although study suggests very high prevalence rate. It may be due to the stigma attached to ear cleaning. Thus it is important that we look for these specific findings associated with ear cleaning so that we can advise against its usage.

This study has some limitations. It was conducted in JDWNRH, the tertiary care center in Bhutan. Although people from all 20 districts come to avail care, a multicentric study would have been more representative of Bhutanese population.

In Conclusion, Bhutan has very high prevalence of ear cleaning irrespective of education level (92.15\%) and many are using dangerously sharp objects to clean them. There are characteristic physical signs associated cotton bud usage as discussed above and their identifications by the health care workers are important to prevent dangerous complications associated with ear cleaning. Proper counseling and public awareness against the use of cotton bud are necessary to prevent such complications.

\section{ACKNOWLEDGEMENTS}

I would like to acknowledge Bhutan Foundation and Khesar Gyalpo University of Medical Science of Bhutan for supporting the study.

I would like to acknowledge the contribution of all ENT staffs in JDWNRH for the conduct of the study.

I would like to thank Dr. Sithar Dorjee for his invaluable insight in the conduct of the study.

\section{REFERENCES}

1. Roland PS, Smith TL, Schwartz SR, Rosenfeld RM, Fayad J, Bopanna B, et al. Clinical practice guideline: Cerumen impaction. Otolaryngol Head Neck Surg. 2008;139:S1-S21. [Full Text | DOI]
2. Olaosun AO. Self-ear-cleaning among educated young adults in Nigeria. J Family Med Prim Care. 2014;3:17-21. [PubMed | Full Text | DOI]

3. Amutta SB, Yunusa MA, Iseh KR, Obembe A, Egili E, Aliyu $\mathrm{D}$, et al. Sociodemographic characteristics and prevalence of self ear cleaning in Sokoto metropolis. Int J Otorhinolaryngol Head Neck Surg. 2013;2:276-9. [Full Text | DOI]

4. Hobson JC, Javy JA. Use and abuse of cotton buds. J R Soc Med. 2005 Aug; 98(8):360-1. [Full Text | DOI]

5. Raman R. Should cotton buds be banned? Trop Doct. 1997;27(4):250. [Full Text | DOI]

6. Ahmed S, Mahinda KH, Khan AA, Iqbal K, Younus I. Cotton bud for ears-friend or foe? Pakistan journal of otolaryngology head and neck surgery. 2009;25:6-8.

7. Reiss M, Reiss G, Pausch NC. Some view points on ear cleaning. Praxis (Bern 1994). 2000 Jul 13;89(27-28):1154-6. [PubMed]

8. Nussinovitch M, Rimon A, Volovitz B, Raveh E, Prais D, Amir J. Cotton-tip applicators as a leading cause of otitis externa. Int J Pediatr Otorhinolaryngol. 2004;68(4):433-5. [Full Text $\mid$ DOI]

9. Smith M, Ilaaf D, Seidman M. Otologic complications of cotton swab use. Laryngoscope. 2012;122(2) :409-11. [Full Text | DOI]

10. McCarter DF, Courtney AU, Pollart SM. Cerumen Impaction. Am Fam Physician. 2007 May 15;75(10):1523-8. [PubMed | Full Text]

11. Kravitz H, Nebus L, Dale D, Laker LH, Gomberg RM, Korach A. Cotton tipped swab:a major cause of ear injury and hearing loss. Clinical Pediatr. 1974;13:965-70. [PubMed | Full Text | DOI]

\section{AUTHORS CONTRIBUTION}

The following authors have made substantial contributions to the manuscript as under:

TRA: Concept, study design, data collection and oversight, critical reviews, research dissemination

SJ: Concept, study design, data analysis, manuscript drafting, critical reviews, research dissemination

Authors agree to be accountable for all respects of the work in ensuring that questions related to the accuracy and integrity of any part of the work are appropriately investigated and resolved.

\section{CONFLICT OF INTEREST}

None

GRANT SUPPORT AND FINANCIAL DISCLOSURE

None 